\title{
Detecting the Stable, Observable and Controllable States of the Human Brain Dynamics
}

\author{
Ehsan Kamrani, Armin N. Foroushani, Mohsen Vaziripour, Mohamad Sawan \\ Electrical Engineering Department, Ecole Polytechnique, Montreal, Canada \\ Email: ehsan.kamrani@ieee.org
}

Received August 13, 2012; revised September 25, 2012; accepted October 6, 2012

\begin{abstract}
A new technique is proposed in this paper for real-time monitoring of brain neural activity based on the balloon model. A continuous-discrete extended Kalman filter is used to estimate the nonlinear model states. The stability, controllability and observability of the proposed model are described based on the simulation and measured clinical data analysis. By introducing the controllable and observable states of the hemodynamic signal we have developed a numerical technique to validate and compare the impact of brain signal parameters affecting on BOLD signal variation. This model increases significantly the signal-to-noise-ratio (SNR) and the speed of brain signal processing. A linear-quadratic regulator (LQR) also has been introduced for optimal control of the model.
\end{abstract}

Keywords: BOLD Signal; Hemodynamics; Controllability and Observability; FNIRS; Brain Imaging; Brain Dynamics

\section{Introduction}

In the brain, real-time monitoring of hemodynamic states and preserving their stability provides a significant mechanism for fast and reliable brain monitoring especially in early detection of seizure and epilepsy or in brainmachine interaction studies. In order to measure the neural activity of the brain the electroencephalography (EEG) and magnetoencephalography (MEG) could be applied for electrophysiological aspects and the functional magnetic resonance imaging (fMRI) and functional near infrared spectroscopy (fNIRS) [1] for metabolic aspects. When the blood oxygenation changes in the brain, it shows that we have a neural activity. So, this is a way to track the neural activity by detecting the hemodynamic changes in the brain. Blood oxygen level-dependent (BOLD) signal shows the brain activity and fMRI and fNIRS use this signal to show this activity. Many experimental observations have provided evidence of the deviation of BOLD from linearity [2-7]. With these observations of nonlinearity of the BOLD response, several researchers have attempted to handle nonlinear characterization for these underlying brain processes. In [3], the linear model of heomodynamic response presented in [4] is extended to cover nonlinear responses using a Volterra series expansion.

At the same time the first compelling model for heomodynamic signal transduction in fMRI was presented in [5], namely the Balloon Model. Several works have recently used this physiological model in the analysis of
fMRI data, in the context of parameter estimation. The work presented in [6] uses the Buxton-Fritson model, where the Buxton's balloon model [7] is added with a damped oscillator to model the blood flow [8]. They used a local linearization transfer function in the Kalman filter methodology, allowing physiological noise in addition to the measurement noise.

The work presented in [8] investigates the above physiological model plus the integrated version of the balloon model [9]. They use a maximum likelihood approach for the model based on the optimization of the parameter estimation, however only the measurement noise is dealt with the system modeling. Models of the underlying hemodynamic and physiologic processes which give rise to the BOLD response have recently been incorporated into a more complete nonlinear system. Hemodynamic responses to neuronal activity are observed experimentally in fMRI data via the BOLD signal, which provides a noninvasive measure of neuronal activity.

Despite the widespread use of functional neuroimaging techniques $[6,7,11,12]$, the physiological changes in the brain that accompanying neural activation are still poorly understood [2-7]. Due to the nonlinear and/or unspecified effects of different parameters on BOLD signal variation, there is no specific criterion to validate and observe the impact of each parameter.

The highly dependency and correlation of neurons processing, metabolic and vascular responses are conceptually well known in time and state space [13], but still the details on the translation between an ensemble of neurons 
firing and the ensuing increase in focal cerebral blood flow is a controversial issue. The most popular model to describe the neural activity according to the data from fMRI, is balloon model, which relates BOLD signal to the blood flow. This model is a nonlinear hemodynamic model and the measurements usually have a noisy behavior. Furthermore, the electromagnetic field produced by neurons is very weak and noisy, so the SNR is very low. No quantified technique has been proposed yet in order to validate and compare the effect of hemodynamic parameters.

We have introduced an efficient hemodynamic state stimulation technique at [14] using fNIRS Data with the Extended Kalman Filter and Bifurcation Analysis of Balloon Model. Here we have used a modified and integrated version of the balloon model [7], using state space system realization to be easily applied in any control system. We prefer to use this particular version of the balloon model since it has many degrees of freedom comparing to the other models $[7,8]$ and can therefore produce a more desired behavior. An extended Kalman filter is applied as a reasonable model to estimate the nonlinear model states and output of the balloon model to extract data from the signal and increase the SNR. By introducing the controllable and observable states of the hemodynamic signal we have also developed a numerical technique to validate and compare the impact of brain signal parameters affecting on BOLD signal variation.

As a consequence, in this paper the proposed model is introduced in Section 2. Section 3 presents the simulation and experimental results following by analysis and discussions on the stability, controllability and observability of the proposed system. A linear-quadratic regulator (LQR) also has been introduced at the end.

\section{Proposed Model}

\subsection{The Extended Balloon Model}

We have introduced an extended balloon model as depicted in Figure 1. The balloon model relates BOLD signal to the blood flow. The balloon model is expanded by a difference in normalized venous out-flow $f_{\text {out }}(v, t)$ and normalized arterial inflow $f_{\text {in }}(t)$. The cerebral blood flow $(\mathrm{CBF})$ is also considered identical to $f_{\text {in }}(t)$ in most works $[9-13,15]$. Conservation of mass then defines the change in the normalized blood volume $v$ in the venous balloon as follow:

$$
\begin{aligned}
& \frac{\mathrm{d} v}{\mathrm{~d} t}=\frac{1}{\tau_{0}}\left(f_{\text {in }}(t)-f_{\text {out }}(v, t)\right) \\
& f_{\text {out }}(t)=v^{1 / \alpha}+\tau_{+/-}\left(\frac{\mathrm{d} v}{\mathrm{~d} t}\right)
\end{aligned}
$$

here $\tau_{0}$ is the mean transit time through the compartment, $\tau_{+}(0-30 \mathrm{~s})$ indicated the viscoelastic time constant (inflation) and $\tau_{-}(0-30 \mathrm{~s})$ is the viscoelastic time constant (deflation).

Equation (1) thereby introduces a fundamental nonlinearity, sufficient to generate all transients of the BOLD response. The variation of the normalized [HbR] concentration $(q)$, can be defined as:

$$
\frac{\mathrm{d} q}{\mathrm{~d} t}=\frac{1}{\tau_{0}}\left(f_{\text {in }}(t) \frac{E(t)}{E_{0}}-f_{\text {out }}(v, t) \frac{q(t)}{v(t)}\right) .
$$

The core of the model is the physical necessity to largely increase $\mathrm{CBF}, f_{\text {in }}(t)$ to achieve a small increase in oxygen delivery. An increase in cerebral blood flow is very closely linked to the underlying neuronal activity [9]. Due to the significant noise induced by measurements we have applied a stochastic hemodynamic system model to describe it. A continuous-discrete extended Kalman filter is used as a reasonable model to estimate the nonlinear states of the balloon model. The Balloon model [7] is an input-state-output nonlinear hemodynamic model with two state variables volume $(v)$ and deoxy-hemoglobin content $(q)$. The input to the system is blood flow $\left(f_{\text {in }}\right)$ and the output is the BOLD signal $(y)$. The BOLD signal is partitioned into an extra and intravascular component, weighted by their respective volumes. These signal components depend on the deoxy-hemoglobin content and render the signal a nonlinear function of $v$ and $q$.

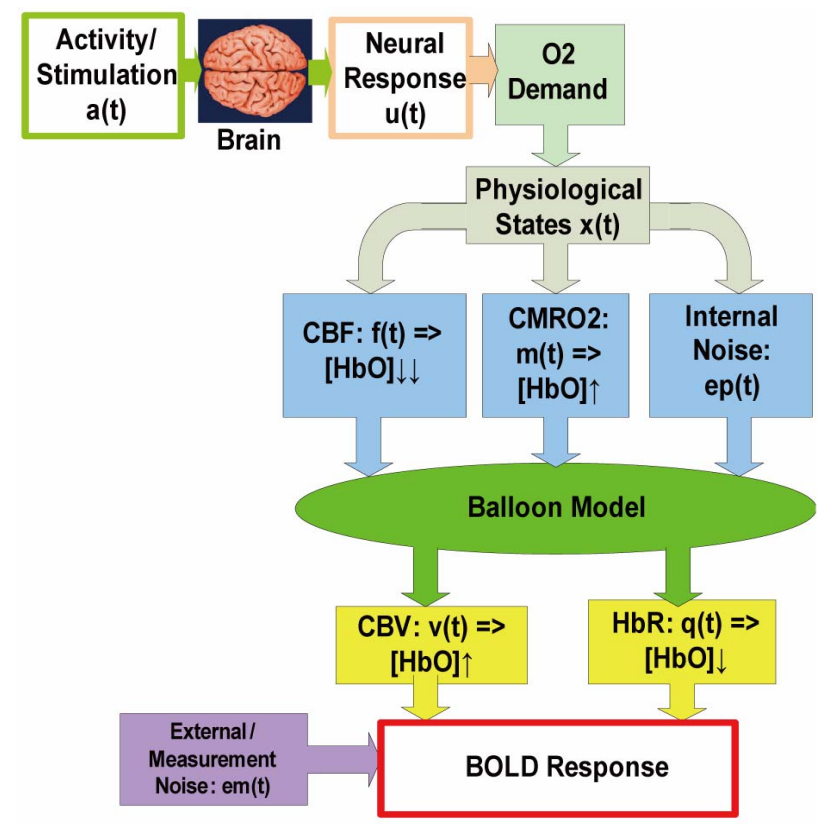

Figure 1. Overview diagram of the applied brain hemodynamic model. 
By extending the model to cover the dynamic coupling of synaptic activity and flow a complete model, relating experimentally induced changes in neuronal activity to BOLD signal, obtains. Here we have considered four different states include: $v$ cerebral blood volume (CBV), $q$ deoxyhaemoglobin content, $s$ flow inducing signal, $f$, $\mathrm{CBF}$. These equations are acquired from the magnetic properties of hemoglobin which is diamagnetic for oxyhemoglobin and paramagnetic for deoxy-hemoglobin. Using the electromagnetic equations around a cylinder and variation with oxygen saturation, the balloon model can be obtained. The neural activity signal $u$ is the input of the model. The mathematical expression of hemodynamic balloon model is as follows:

$$
\begin{gathered}
\dot{v}=\frac{1}{\tau_{0}}\left(f-v^{(1 / \alpha)}\right), \\
\dot{q}=\frac{1}{\tau_{0}}\left(\frac{f\left(1-\left(1-E_{0}\right)^{\frac{1}{f}}\right)}{E_{0}}-\frac{q}{v^{1-\frac{1}{\alpha}}}\right), \\
\dot{s}=\varepsilon u-\frac{1}{\tau_{s}} s-\frac{1}{\tau_{f}}(f-1) ; \quad \dot{f}=s .
\end{gathered}
$$

And output which is BOLD signal is:

$$
y=V_{0}\left(a_{1}(1-q)-a_{2}(1-v)\right) .
$$

We can measure a new parameter $m(t)$, which is CMRO2 normalized to baseline too:

$$
m(t)=\frac{f\left(1-\left(1-E_{0}\right)^{\frac{1}{f}}\right)}{E_{0}} .
$$

In this model $E_{0}$ is baseline oxygen extraction fraction, $V_{0}$ is baseline blood volume, $a_{1}$ is weight for deoxy $\mathrm{Hb}$ change and $a_{2}$ is the weight for blood volume. $\tau_{0}$ is the mean transit time of the venous compartment, $\alpha$ is the stiffness component of the balloon model, $\tau_{s}$ is the signal decay time constant, $\tau_{f}$ is the autoregulatory time constant, and $\varepsilon$ is the neuronal efficacy. Now, we can describe the state space equations as a nonlinear dynamic system. The state of the system is a vector:

$$
\frac{\partial F}{\partial x}=\left[\begin{array}{ccc}
-\frac{1}{\tau_{0} \alpha} v^{(1 / \alpha)-1} & 0 & 0 \\
-\frac{q}{\tau_{0}}\left(\frac{1-\alpha}{\alpha}\right) v^{(1 / \alpha)-2} & -\frac{v^{(1 / \alpha)-1}}{\tau_{0}} & 0 \\
0 & 0 & -\frac{1}{\tau_{s}} \\
0 & 0 & 1
\end{array}\right.
$$

$$
x=\left[\begin{array}{llll}
v & q & s & f
\end{array}\right]^{T}
$$

\subsection{The Extended Kalman Filter}

Extended Kalman filter is a nonlinear version of Kalman filter using for nonlinear dynamic systems, applied to estimate the states of balloon model. The nonlinear stochastic dynamical system is described by following state space equation:

$$
\begin{aligned}
& \dot{x}(t)=F(x(t), u(t))+G w(t) ; w(t) \sim N(0, Q), \\
& y_{k}=H\left(x_{k}\right)+v_{k} ; v_{k} \sim N(0, R) .
\end{aligned}
$$

This model includes perturbation and measurement noise, because of weak signal of fMRI. In this state space equation, $x(t)$ is the state which is dependent on time, $u(t)$ is input stimulus, $w(t)$ is the perturbation noise (a white noise) which has mean 0 and variance $Q . v_{k}$ is measurement noise which is a white noise with mean 0 and variance $R$. The $x_{k}=x\left(t_{k}\right)$ and $w(t), v_{k}$ are independent Gaussian sequences having the following properties:

$$
\begin{array}{ll}
E[w(t)]=E\left[v_{k}\right]=0 ; & E\left[w(t) w(t)^{T}\right]=Q ; \\
E\left[v_{k} v_{k}^{T}\right]=R ; & E\left[w(t) v_{k}^{T}\right]=0 ; \\
E\left[w(t) x_{k}^{T}\right]=0 ; & E\left[v_{k} x_{k}^{T}\right]=0 .
\end{array}
$$

The prediction is established as:

$$
\begin{gathered}
\left\{\begin{array}{l}
\hat{\mathbf{x}}_{k+1 / k}=F\left(\hat{\mathbf{x}}_{k}, u_{k}\right) \\
\hat{\mathbf{x}}_{k}=\hat{\mathbf{x}}\left(t_{k}\right) \\
\hat{\mathbf{x}}_{k+1 / k}=\hat{\mathbf{x}}\left(t_{k+1}\right),
\end{array}\right. \\
\left\{\begin{array}{l}
\sum_{k+1 / k}=\frac{\partial F}{\partial \mathbf{x}}\left|\hat{\mathbf{x}}\left(t_{k}\right) \sum_{k} \frac{\partial F^{T}}{\partial x}\right| \hat{\mathbf{x}}\left(t_{k}\right)+G Q G^{\mathrm{T}} \\
\sum_{k+1 / k}=\sum\left(t_{k}+1\right) \\
\sum_{k}=\sum\left(t_{k}\right) .
\end{array}\right.
\end{gathered}
$$

The Jacobian matrix and $\frac{\partial H}{\partial x}$ are defined as follow:

$$
\left.\begin{array}{c}
\frac{1}{\tau_{0}} \\
\frac{1}{\tau_{0} E_{0}}\left(1-\left[\left(1-E_{0}\right)^{1 / f}-\frac{1}{f}\left(1-E_{0}\right)^{1 / f} \ln \left(1-E_{0}\right)\right]\right), \\
-\frac{1}{\tau_{f}} \\
0
\end{array}\right]
$$




$$
\frac{\partial H}{\partial x}=\left[\begin{array}{llll}
a_{2} V_{0} & -a_{1} V_{0} & 0 & 0
\end{array}\right] .
$$

The input signal $u$ in the balloon model is the neural activity and it is created by a square stimulus signal $a(t)$. The relation between the neural activity and the stimulus signal can be stated by:

$$
\begin{aligned}
& u(t)=a(t)-I(t), \\
& \frac{\mathrm{d} I}{\mathrm{~d} t}=\frac{k u(t)-I(t)}{\tau_{u}},
\end{aligned}
$$

where $a(t)$ is the stimulus step signal and $I(t)$ is an inhibitory feedback signal and $k$ is a gain factor. $\tau_{u}$ is a time constant. So, first the neural activity $u(t)$ will be produced from $a(t)$ and then use neural activity as an input to the balloon model.

\section{Simulation and Experimental Results}

\subsection{Simulation and Measurement Setup}

The balloon model is implemented in Simulink and a reasonable neural activity input is produced. Then the output is plotted as a BOLD signal. A white noise is added to this signal in order to mimic a noisy BOLD signal. Using proposed extended Kalman filter, the output due to the noisy signal follows the measurements (Figure 2).

The proposed system is verified using measured clinical data also plotted in Figure 3. The extended Kalman filter is used to estimate the BOLD signal. Figure 4 shows the simulated, measured, and the estimated BOLD signals. The effect of $\tau_{s}$ is very important especially on the time of reaching to steady state. For higher $\tau_{s}$ the system will reach to steady state later and, so if our purpose is to reach to steady state condition, we should have a small $\tau_{s}$. As it is supposed when the initialized variance $\left(\Sigma_{0}\right)$ increases that is the initial conditions are unknown and it causes that the contribution of measurements in the update equation increases which results the faster convergence. For higher $\Sigma_{0}$ especially when the system starts, Kalman gain is more than the case of the lower $\Sigma_{0}$. The convergence is slower when $\Sigma_{0}$ is low which means we trust to the initial values. Due to the relationship between $R$ and Kalman gain, when the level of the noise at the measured value increases ( $R$ increases) the gain will decrease and the contribution of measurement in the update equation will decrease. Therefore slower convergence is a direct result of the higher noise level at the output.

\subsection{Bifurcation Analysis}

Bifurcation analysis investigates the stability of the system under change of parameters. Thus, it is important to first investigate nonlinear stability of the system and then use MatCont for Bifurcation analysis.

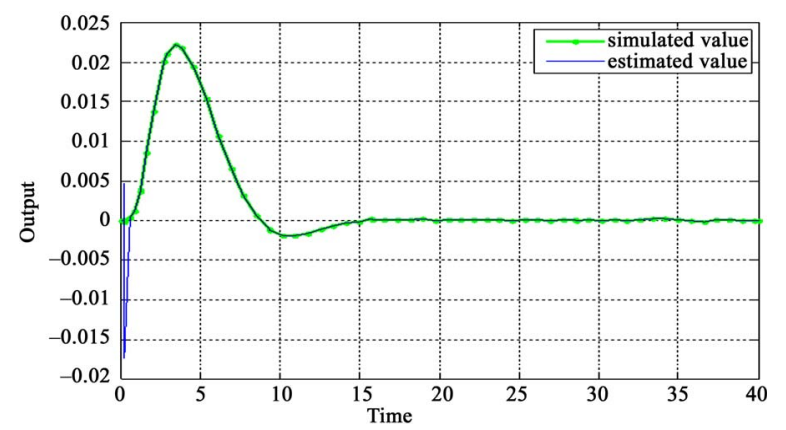

(a)

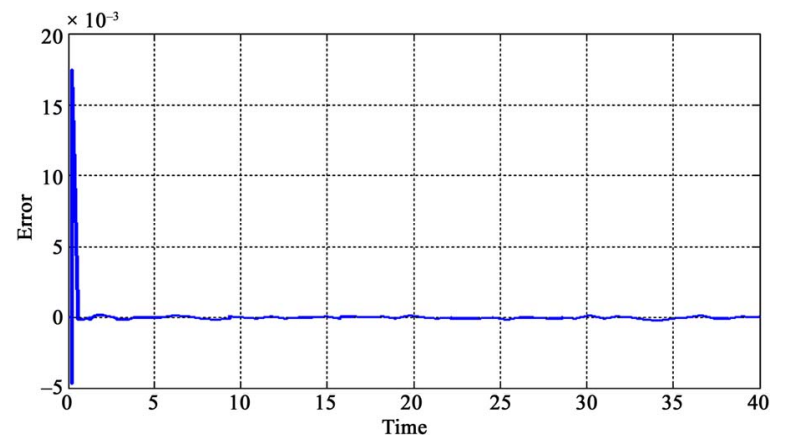

(b)

Figure 2. Dynamics of the hemodynamic model: (a) The BOLD signal and its estimation; (b) The estimation error of BOLD signal.

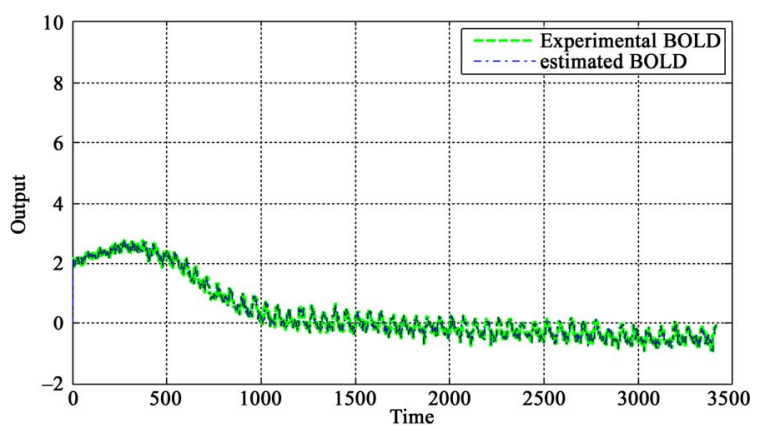

(a)

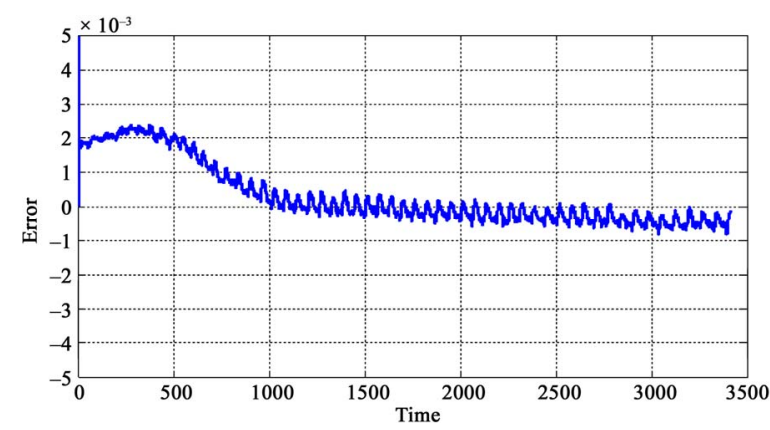

(b)

Figure 3. (a) Real experimental measurement of output data and its estimation; (b) The Experimental error. 


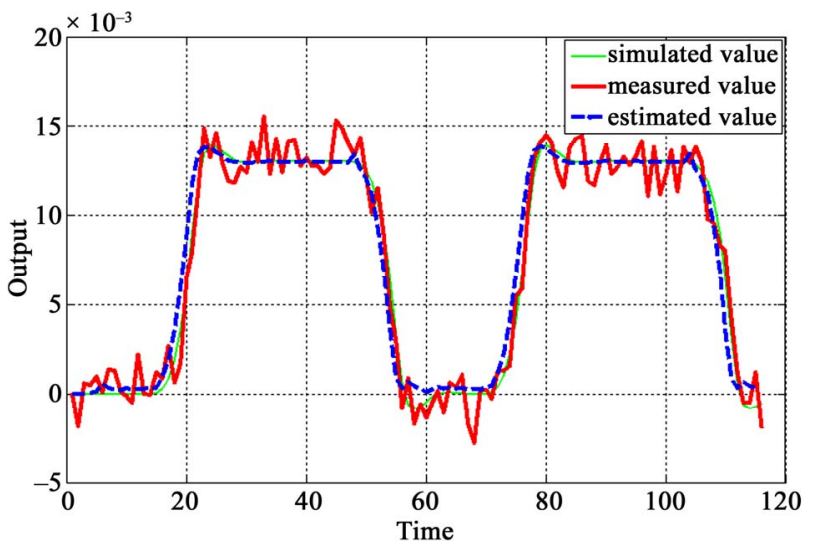

(a)

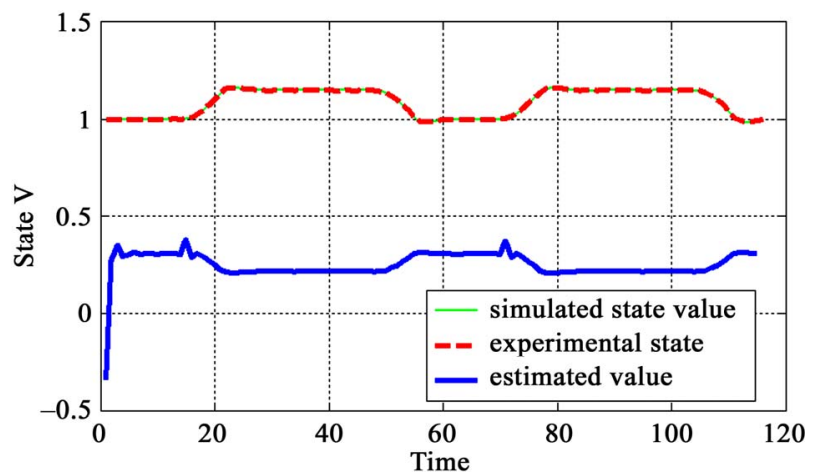

(b)

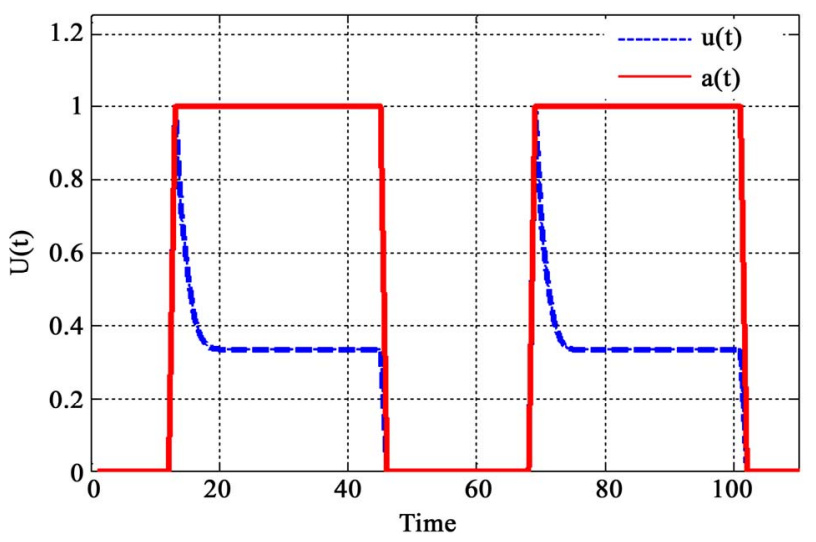

(c)

Figure 4. (a) The simulated; (b) Measured; (c) The estimated BOLD signals.

For bifurcation analysis first the equilibrium point should be calculated as follow:

$$
\left\{\begin{array}{l}
v=\left(1+\varepsilon u \tau_{f}\right)^{\alpha} \\
q=\left(1+\varepsilon u \tau_{f}\right)^{\alpha}\left(\frac{1-\left(1-E_{0}\right)^{\frac{1}{1+\varepsilon u \tau_{f}}}}{E_{0}}\right) . \\
s=0 \\
f=1+\varepsilon u \tau_{f}
\end{array}\right.
$$

As we see the important parameters for the equilibrium point are $E_{0}, \varepsilon, \tau_{f}, \alpha$ and we have investigated their effects in the bifurcation analysis. Now, we put this equilibrium point in the Jacobian matrix and then find the eigenvalues of that matrix to investigate the nonlinear stability of balloon model for different parameters.

The eigenvalues of the Jacobian matrix in this case are:

$$
\left\{\begin{array}{l}
\lambda_{1}=-\frac{1}{\tau_{0}}\left(1+\varepsilon u \tau_{f}\right)^{1-\alpha} \\
\lambda_{2}=-\frac{1}{\tau_{0} \alpha}\left(1+\varepsilon u \tau_{f}\right)^{1-\alpha} \\
\lambda_{3,4}=\frac{\left(-1 / \tau_{s}\right) \pm \sqrt{\left(1 / \tau_{s}\right)^{2}-4\left(1 / \tau_{f}\right)}}{2}
\end{array} .\right.
$$

As we see all of the eigen-values are negative and this system for every choice of parameters is always stable. This is very interesting achievement regarding to the stability characteristics of the proposed system to describe the hemodynamic parameters. These eigenvalues depend on $\varepsilon, \tau_{s}, \tau_{0}, \tau_{f}, \alpha$ and independent of $E_{0}$. For the bifurcation analysis, in this paper MatCont is used to analyze stability of the system with change of different effective parameters of the system. Figure 4 compares the simulated and estimated BOLD signals with the measurement data.

\subsection{Stability}

Bifurcation analysis shows that the nonlinear stability of balloon model is always guarantied. Here the stability is also represented based on the linearization of the balloon model. This is a simpler model of stability when all of the eigenvalues of the system $\left(\lambda_{1}, \lambda_{2}, \lambda_{3}, \lambda_{4}\right)$ are negative. In this situation, the system is linearizable and it is possible to linearize the state space equation and then use the definition of stability, observability, and controllability in the linear case. The linear model is in the form of:

$$
\left\{\begin{array}{l}
\dot{x}=A x+B u \\
y=C x+D u
\end{array}\right.
$$

where:

$$
\begin{aligned}
& A=\left.\frac{\partial F(x(t), u(t))}{\partial x}\right|_{x_{e}, u_{e}} ; \\
& B=\left.\frac{\partial F(x(t), u(t))}{\partial u}\right|_{x_{e}, u_{e}} ; \\
& C=\left.\frac{\partial y}{\partial x}\right|_{x_{e}, u_{e}} ; \quad D=\left.\frac{\partial y}{\partial u}\right|_{x_{e}, u_{e}} .
\end{aligned}
$$

and $\left(x_{e}, u_{e}\right)$ is the equilibrium point. 


$$
A=\left[\begin{array}{cccc}
-\frac{1}{\tau_{0} \alpha} & 0 & 0 & \frac{1}{\tau_{0}} \\
-\frac{1-\alpha}{\tau_{0} \alpha} & -\frac{1}{\tau_{0}} & 0 & \frac{1}{\tau_{0} E_{0}}\left(1-\left(1-E_{0}\right)\left(1-\ln \left(1-E_{0}\right)\right)\right) \\
0 & 0 & -\frac{1}{\tau_{s}} & -\frac{1}{\tau_{f}} \\
0 & 0 & 1 & 0
\end{array}\right]
$$

The eigenvalues of $A$ are:

$$
\begin{aligned}
& \lambda_{1}=-\frac{1}{\tau_{0}} ; \quad \lambda_{2}=-\frac{1}{\tau_{0} \alpha} ; \\
& \lambda_{3,4}=\frac{\left(-1 / \tau_{s}\right) \pm \sqrt{\left(1 / \tau_{s}\right)^{2}-4\left(1 / \tau_{f}\right)}}{2} .
\end{aligned}
$$

All of these eigenvalues are negative and equal to the case of nonlinear stability analysis when $u=0$. So, the system is stable. The Bode diagram and the Root-Locus of the open-loop and closed-loop systems are shown in Figure 5.

\subsection{Controllability and Observability}

The linear and nonlinear controllability and observability has been investigated at this section. For linear controllability and observability based on the controllability matrix $\left[\mathrm{B} \mathrm{ABA}^{2} \mathrm{BA}{ }^{3} \mathrm{~B}\right]$ and observability index $\left[\mathrm{CCACA}^{2} \mathrm{CA}^{3}\right]^{\mathrm{T}}$, we can determine their determinant to investigate if the system is controllable and observable or not. The determinant of each matrix is as followed:

$$
\begin{aligned}
& \operatorname{det}\left(\left[B A B A^{2} B A^{3} B\right]\right)=\frac{1-E_{0}-\alpha+E_{0} \alpha}{E_{0} \alpha \tau_{0}{ }^{3}} \log \left(1-E_{0}\right) \\
& \operatorname{det}\left(\left[\mathrm{C} \mathrm{CACA}^{2} \mathrm{CA}^{3}\right]^{\mathrm{T}}\right) \\
& =f\left(v_{0}, a_{1}, a_{2}, \alpha, \tau_{0}, \tau_{f}, \tau_{s}, E_{0}\right) .
\end{aligned}
$$

For nonlinear controllability and observability, the nonlinear balloon model is directly investigated. For nonlinear controllability we have:

$$
\begin{aligned}
\operatorname{det}(C)=|C|= & {\left[\left(1-\frac{1}{\alpha}\right) \frac{1}{\tau_{0}^{2}} v^{(1 / \alpha)-1}\right] } \\
& \times\left[\frac{\varepsilon}{\tau_{0} E_{0}}\left[1-\left(1-E_{0}\right)^{1 / f}\left(1-\frac{\ln \left(1-E_{0}\right)}{f}\right)\right]\right] \\
& +\frac{q}{\tau_{0}^{3}}\left(1-\frac{1}{\alpha}\right) v^{(1 / \alpha)-2} \\
& -\frac{1}{\tau_{0}^{2} E_{0}} \frac{\left(1-E_{0}\right)^{1 / f}\left(\ln \left(1-E_{0}\right)\right)^{2}}{f^{3}} \mathrm{~S} .
\end{aligned}
$$

$0 \quad 0] ; D=0$.

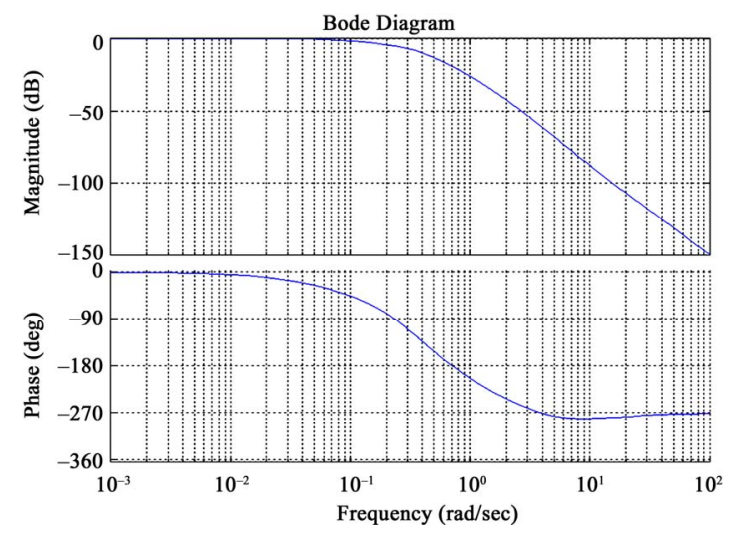

(a)

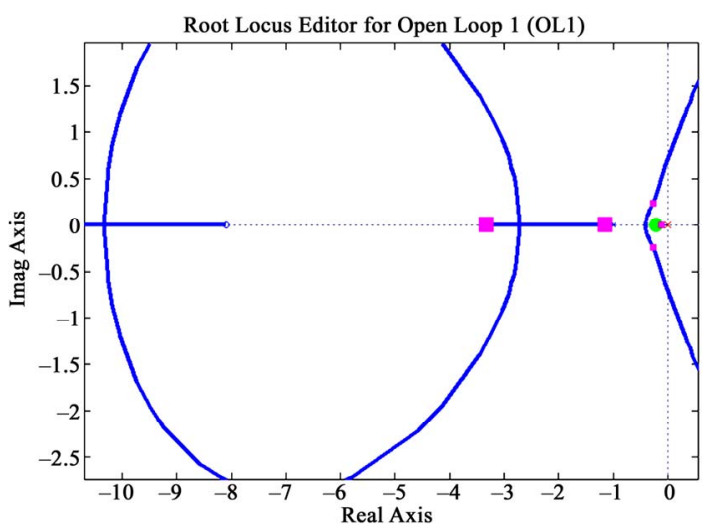

(b)

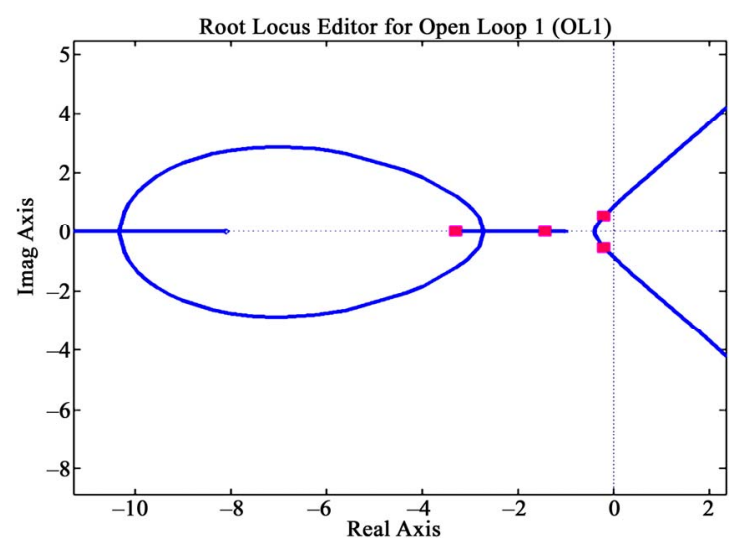

(c)

Figure 5. Stability analysis using (a) Bode diagram; (b) Openloop root locus; (c) Closed-loop root locus. 
For nonlinear observability we have:

$$
\begin{gathered}
L(x)=\left[\begin{array}{l}
L_{f}^{0}(h) \\
L_{f}^{1}(h) \\
L_{f}^{2}(h) \\
L_{f}^{3}(h)
\end{array}\right]=\left[\begin{array}{l}
h \\
h \\
\ddot{h} \\
\dddot{h}
\end{array}\right], \\
A=\left[\begin{array}{llll}
a_{11} & a_{12} & a_{13} & a_{14} \\
a_{21} & a_{22} & a_{23} & a_{24} \\
a_{31} & a_{32} & a_{33} & a_{34} \\
a_{41} & a_{42} & a_{43} & a_{44}
\end{array}\right] .
\end{gathered}
$$

where:

$$
\begin{aligned}
& a_{13}=a_{14}=a_{23}=0 ; \quad a_{11}=\frac{\partial h}{\partial v}=a_{2} V_{0} ; \\
& a_{12}=\frac{\partial h}{\partial q}=-a_{1} V_{0} ; \quad a_{13}=\frac{\partial h}{\partial S}=0 ; \\
& a_{14}=\frac{\partial h}{\partial f}=0 ; \quad a_{21}=\frac{\partial \dot{h}}{\partial v} ; \quad a_{22}=\frac{\partial \dot{h}}{\partial q} ; \\
& a_{23}=\frac{\partial \dot{h}}{\partial s}=0 ; \quad a_{24}=\frac{\partial \dot{h}}{\partial f} ; \quad a_{31}=\frac{\partial \ddot{h}}{\partial v} ; \\
& a_{32}=\frac{\partial \ddot{h}}{\partial q} ; \quad a_{33}=\frac{\partial \ddot{h}}{\partial s} ; \quad a_{34}=\frac{\partial \ddot{h}}{\partial f} ; a_{41}=\frac{\partial \dddot{h}}{\partial v} ; \\
& a_{42}=\frac{\partial \dddot{h}}{\partial q} ; \quad a_{43}=\frac{\partial \dddot{h}}{\partial s} ; \quad a_{44}=\frac{\partial \dddot{h}}{\partial f} .
\end{aligned}
$$

The observability and controllability of linear and nonlinear systems depend on all previously introduced parameters, so they have been calculated in equilibrium point for all of these parameters. The calculation results show that for these values the system is controllable and observable.

\subsection{The LQR Controller Design}

As the system is controllable we can design an LQR controller. So, a full state feedback $u=-k x$ with a proper gain vector $k$ can effectively control the system in a neighborhood of the equilibrium point. We consider the output $y=V_{0}\left(a_{2} v-a_{1} q\right)$ to be matched with the experimental results. The input, output and the states of the system are shown in Figures 6 and 7, when the LQR controller is applied.

\section{Conclusions}

A new model for real-time monitoring of brain neural activity is proposed in this paper based on the balloon model. The stability, controllability and observability of the proposed model are described based on the simulation and measured clinical data analysis. By introducing the controllable and observable states of the hemody- namic signal we have developed a numerical technique to validate and compare the impact of brain signal parameters affecting on BOLD signal variation. This model increases significantly the SNR and the speed of brain signal processing. Up to our knowledge this is the first work on evaluation of these control parameters and introducing their practical impacts on clinical application. Surprisingly we realized that the system is always stable independent from any variation in blood flow and $\mathrm{HbR} / \mathrm{HbO}$ variation.

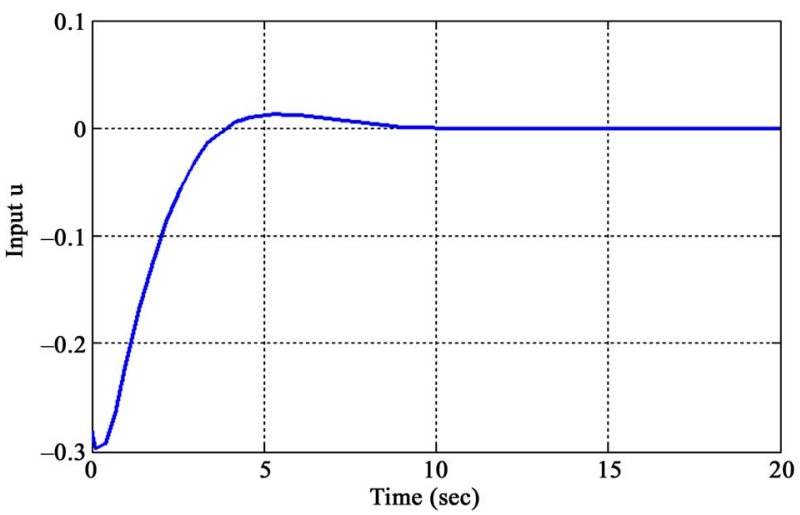

(a)

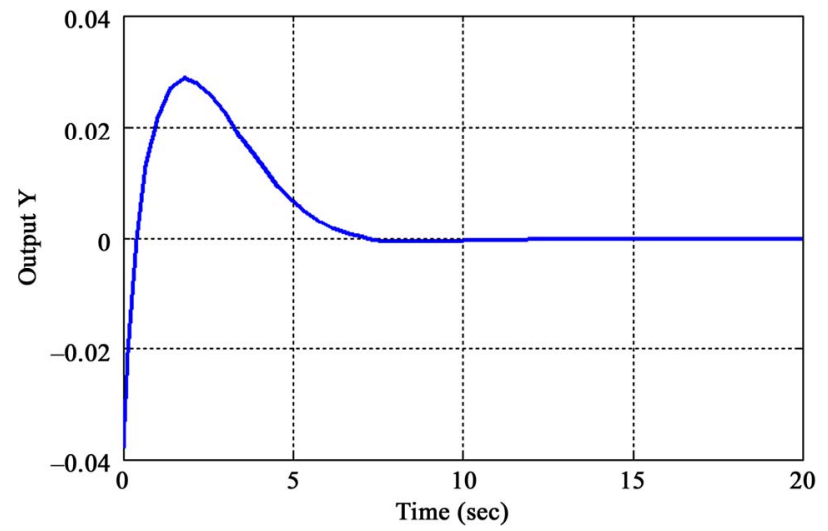

(b)

Figure 6. Input (a) and output (b) signals of the LQR Controller.

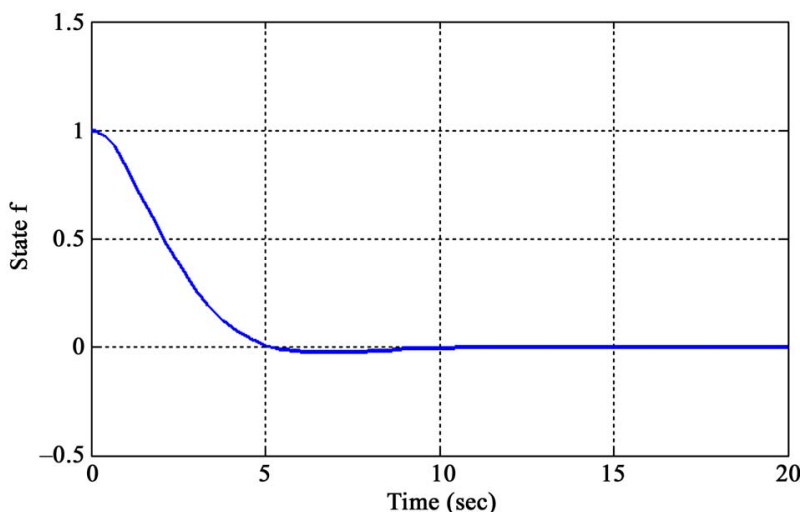

(a) 


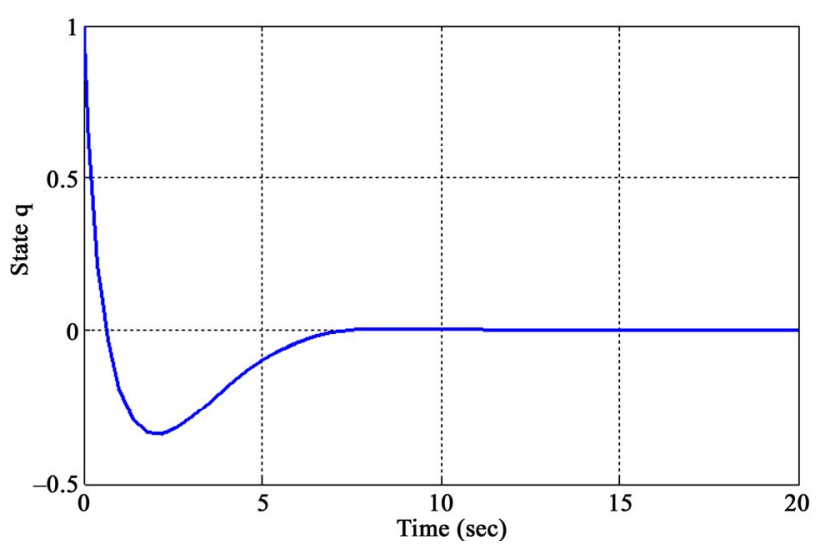

(b)

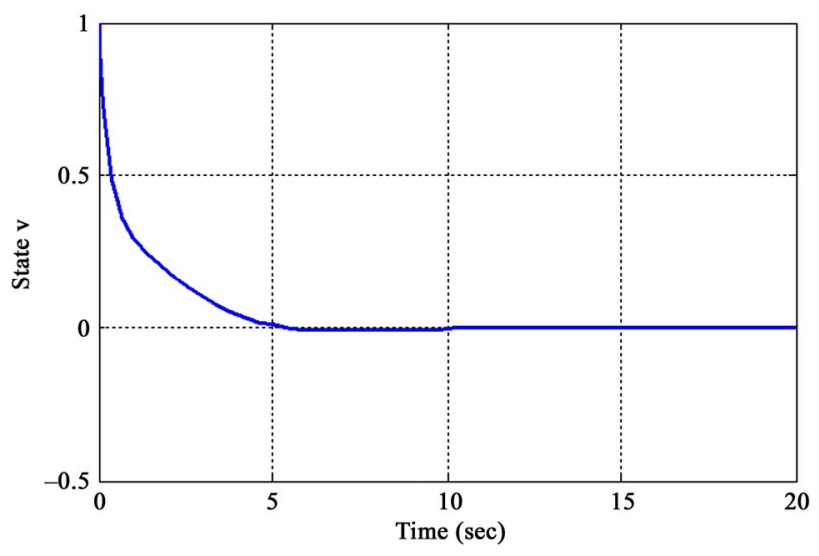

(c)

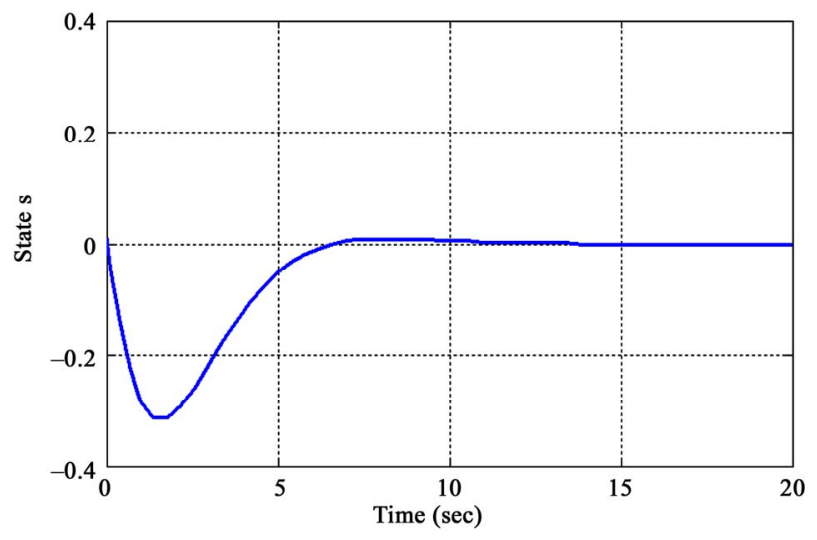

(d)

Figure 7. States of the system when using LQR Control.

The observability and controllability characteristics are introduced as significant factors to be considered as an evaluation tool to verify the preference of different hemodynamic factors. The preferred factors then can be considered based on their specified priority for further diagnosis and monitoring in clinical applications. This model can also be efficiently applied in any monitoring and control platform include brain and for study of hemodynamic and brain imaging modalities such as pulse-oximetry and fNIRS.

\section{REFERENCES}

[1] E. Kamrani and M. Sawan, "Fully Integrated CMOS Avalanche Photodiode and Distributed-Gain TIA for CW-FNIRS," Proceeding of the IEEE Biomedical Circuits and Systems Conference, San Diego, 19 December 2011, pp. 317- 320 . doi: 10.1109/BioCAS.2011.6107791

[2] T. Obata, T. T. Liu, K. L. Miller, W.-M. Luh, E. C. Wong, L. R. Frank and R. B. Buxton, "Dispercencies between BOLD and Flow Dynamicsin Primary and Supplementary Motor Areas: Application of the Balloon Model to the Interpretation of BOLD Transients," NeuroImage, Vol. 21, No. 1, 2004, pp. 144-153. doi:10.1016/j.neuroimage.2003.08.040

[3] K. J. Fritson, O. Josephs, G. Rees and R. Turner, "Nonlinear Eventrelated Responses in FMRI," Magnetic Resonance in Medicine, Vol. 39, No. 1, 1998, pp. 41-52. doi:10.1002/mrm.1910390109

[4] K. J. Fritson, P. Jezzard, R. Turner, et al., "Analysis of Functional MRI Time Series," Human Brain Mapping, Vol. 1, No. 1, 1994, pp. 153-171.

[5] N. K. Logothetis, J. Pauls, M. Augath, T. Trinath and Oeltermann, "Neurophysiological Investigation of the Basis of the FMRI Signal," Nature, Vol. 412, No. 6843, 2001, pp. 150-157. doi: $10.1038 / 35084005$

[6] J. Daunizeau, S. J. Kiebel and K. J. Friston, "Dynamic Causal Modelling of Distributed Electromagnetic Responses," NeuroImage, Vol. 47, No. 2, 2009, pp. 590-601. doi:10.1016/j.neuroimage.2009.04.062

[7] R. Buxton, E. Wong and L. Frank, "Dynamics of Blood Flow and Oxygenation Changes during Brain Activation: The Balloon Model," Magnetic Resonance in Medicine, Vol. 39, No. 6, 1998, pp. 855-864. doi: $10.1002 / \mathrm{mrm} .1910390602$

[8] Y. Kong, et al., "A Model of the Dynamic Relationship between Blood Flow and Volume Changes during Brain Activation," Journal of Cerebral Blood Flow \& Metabolism, Vol. 24, No. 12, 2004, pp. 1382-1392. doi:10.1097/01.WCB.0000141500.74439.53

[9] K. J. Friston, A. Mechelli, R. Turner and C. J. Price, "Nonlinear Responses in FMRI: The Balloon Model, Volterra Kernels, and Other Hemodynamics," NeuroImage, Vol. 12, No. 4, 2000, pp. 466-477. doi:10.1006/nimg.2000.0630

[10] T. Deneux and O. Faugeras, "Using Nonlinear Models in FMRI Data Analysis: Model Selection and Activation Detection," NeuroImage, Vol. 32, No. 4, 2006, pp. 16691689. doi:10.1016/j.neuroimage.2006.03.006

[11] I. T. Hettiarachchi, P. N. Pathirana and P. Brotchie, "A State Space Based Approach in Non-Linear Hemodynamic Response Modeling with FMRI Data," IEEE Conference on Engineering in Medicine and Biology Society, Buenos Aires, 11 November 2010, pp. 2391-2394. doi:10.1109/IEMBS.2010.5627400

[12] R. B. Buxton, K. Uludağ, D. J. Dubowitz and T. T. Liu, "Modeling the Hemodynamic Response to Brain Activation," NeuroImage, Vol. 23, Suppl. 1, 2004, pp. S220-S233. doi:10.1016/j.neuroimage.2004.07.013 
[13] F. Javed, et al., "Recent Advances in the Monitoring and Control of Haemodynamic Variables during Haemodialysis: A Review," Physiological Measurement, Vol. 33, No. 1, 2012, pp. R1-R31. doi:10.1088/0967-3334/33/1/R1

[14] E. Kamrani, A. N. Foroushani, M. Vaziripour and M. Sawan, "Efficient Hemodynamic States Stimulation Using FNIRS Data with the Extended Kalman Filter and Bifurcation Analysis of Balloon Model," Journal of Bio- medical Science and Engineering, Vol. 5, No. 11, 2012, pp. 609-628. doi:10.4236/jbise.2012.511076

[15] J. Steinbrink, A. Villringer, F. Kempf, D. Haux, S. Boden and H. Obrig, "Illuminating the BOLD Signal: Combined FMRI-FNIRS Studies," Magnetic Resonance Imaging, Vol. 24, No. 4, 2006, pp. 495-505.

doi:10.1016/j.mri.2005.12.034 